\title{
Under What Condition Do We Get Improved Equalization Performance in the Residual ISI with Non-Biased Input Signals Compared with the Biased Version
}

\author{
Monika Pinchas \\ Department of Electrical and Electronic Engineering, Ariel University, Ariel, Israel \\ Email: monika.pinchas@gmail.com
}

Received 27 January 2015; accepted 1 April 2015; published 2 April 2015

Copyright (C) 2015 by author and Scientific Research Publishing Inc.

This work is licensed under the Creative Commons Attribution International License (CC BY).

http://creativecommons.org/licenses/by/4.0/

(c) (i) Open Access

\begin{abstract}
Recently, closed-form approximated expressions were obtained for the residual Inter Symbol Interference (ISI) obtained by blind adaptive equalizers for the biased as well as for the non-biased input case in a noisy environment. But, up to now it is unclear under what condition improved equalization performance is obtained in the residual ISI point of view with the non-biased case compared with the biased version. In this paper, we present for the real and two independent quadrature carrier case a closed-form approximated expression for the difference in the residual ISI obtained by blind adaptive equalizers with biased input signals compared with the non-biased case. Based on this expression, we show under what condition improved equalization performance is obtained from the residual ISI point of view for the non-biased case compared with the biased version.
\end{abstract}

\section{Keywords}

Residual ISI, Blind Equalization, Blind Deconvolution

\section{Introduction}

We consider a blind deconvolution problem in which we observe the output of an unknown, possibly nonminimum phase, linear system from which we want to recover its input using an adjustable linear filter (equalizer) [1]-[5]. The problem of blind deconvolution arises comprehensively in various applications such as digital communications, seismic signal processing, speech modeling and synthesis, ultrasonic nondestructive evaluation and 
image restoration [1] [2] [6]. The above mentioned possibly nonminimum phase, linear system may be considered for instance as a channel in the communication area. According to [3] [7] [8], the channel is not ideal due to reflections and delays caused by the physical environment such as ground, buildings and cables. Those reflections and delays cause distortion of the received signal which is referred as ISI [3] [9]. Thus, a blind adaptive equalizer may be used to remove the unwanted ISI of the system to produce the source signal [10]-[14]. According to [1][3] [15], the equalization performance depends on the nature of the chosen equalizer (on the memoryless nonlinearity situated at the output of the equalizer's filter), on the channel characteristics, on the added noise, on the step-size parameter used in the adaptation process, on the equalizer's tap length and on the input signal statistics. Fast convergence speed and reaching a residual ISI where the eye diagram is considered to be open (for the communication case) are the main requirements from a blind equalizer [1]-[3] [15]. Fast convergence speed may be obtained by increasing the step-size parameter [1]-[3] [15]. But, increasing the step-size parameter may lead to a higher residual ISI which may not meet any more the system's requirements [1]-[3] [15]. Up to recently, we used time consuming simulation for performance assessment. Recently, closed-form approximated expressions were obtained for the residual ISI valid for the noiseless and unbiased input signal case [15], noisy and unbiased input signal case [1], noisy and unbiased input signal case but where the gain between the equalized output and input signal is equal or less than one [16] and for the noisy and biased input signal case [3]. But, up to now it is unclear under what condition improved equalization performance is obtained in the residual ISI point of view with the non-biased case compared with the biased version.

In this paper, we derive for the real and two independent quadrature carrier case a closed-form approximated expression for the difference in the residual ISI obtained by blind adaptive equalizers with biased input signals compared with the non-biased case. This expression depends on the step-size parameter, equalizer's tap length, input signal statistics, channel power and signal to noise ratio (SNR). In addition, this expression is valid for blind adaptive equalizers where the error fed into the adaptive mechanism, which updates the equalizer's taps, can be expressed as a polynomial function of order three of the equalized output and where the gain between the input and equalized output signal is equal to one as is in the case of Godard's algorithm [17]. Based on this new derived expression we show under what condition improved equalization performance is obtained from the residual ISI point of view for the non-biased case compared with the biased version.

The paper is organized as follows. After having described the system under consideration in Section II, the condition for which improved equalization performance is obtained from the residual ISI point of view for the non-biased case compared with the biased version is introduced in Section III. In Section IV simulation results are presented and the conclusion is given in Section $\mathrm{V}$.

\section{System Description}

The system under consideration is the same system as shown in [1]-[3] [15] and [16]. Thus, we recall from [3] the block diagram of the system illustrated in Figure 1. We adapt in the following most of the assumptions made in [3]:

1) The input sequence $x[n]$ represents a two independent biased or unbiased quadrature carriers case constellation input where $x_{r}[n]$ and $x_{i}[n]$ are the real and imaginary parts of $x[n]$ respectively.

2) The mean of the input sequence $x[n]$ is $E[x[n]]$, where $E[\cdot]$ is the expectation operator and $\tilde{x}[n]=x[n]-E[x[n]]$.

3) $E\left[x_{r}[n]\right]=E\left[x_{i}[n]\right]$.

4) The unknown channel $h[n]$ is modeled as a non-minimum phase FIR filter, which has zeros far from the unit circle.

5) $c[n]$ is a tap-delay line.

6) The noise $w[n]$ is an added Gaussian white noise with zero mean and consists of $w[n]=w_{r}[n]+j w_{i}[n]$ where $w_{r}[n]$ and $w_{i}[n]$ are the real and imaginary parts of $w[n]$ respectively as well as $w_{r}[n]$ and $w_{i}[n]$ are independent. Both $w_{r}[n]$ and $w_{i}[n]$ have zero mean and their variances are denoted as: $\sigma_{w_{r}}^{2}=E\left[w_{r}^{2}[n]\right], \quad \sigma_{w_{i}}^{2}=E\left[w_{i}^{2}[n]\right]$. 


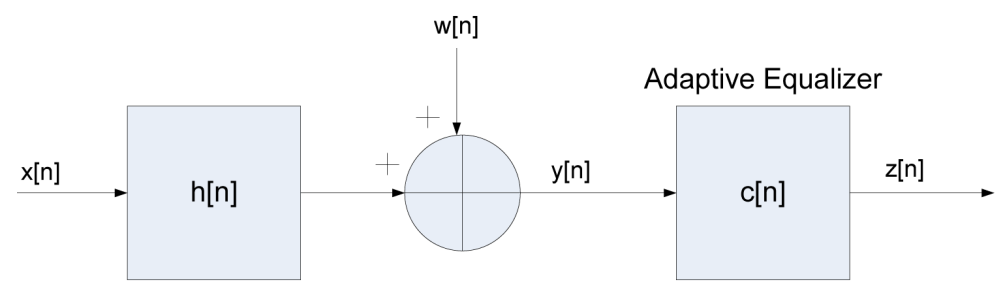

Figure 1. Block diagram of a baseband communication system.

7) The variance of $w[n]$ is denoted as $E\left[w[n] w^{*}[n]\right]=\sigma_{w}^{2}$ where $\sigma_{w}^{2}=2 \sigma_{w_{i}}^{2}=2 \sigma_{w_{r}}^{2}$ and $(\cdot)^{*}$ is the conjugate operation on $(\cdot)$.

The transmitted sequence $x[n]$ is sent through the channel $h[n]$ and is interfered with noise $w[n]$. Therefore, the equalizer's input sequence $y[n]$ may be written as:

$$
y[n]=x[n] * h[n]+w[n]
$$

where "*" denotes the convolution operation. The equalizer's output sequence may be written as:

$$
z[n]=y[n] * c[n]=x[n]+p[n]+\tilde{w}[n]
$$

where $p[n]$ is the convolutional noise that arises due to the use of non-ideal equalizer's coefficients (blind equalization) instead of the ideal set and $\tilde{w}[n]=w[n] * c[n]$. The equalizer's update mechanism is defined by:

$$
\underline{c}[n+1]=\underline{c}[n]-\mu \frac{\partial F[n]}{\partial \tilde{z}[n]} \underline{y}^{*}[n]
$$

where $\mu$ is the equalizer's step-size, $\underline{c}[n+1]$ and $\underline{c}[n]$ are the next and current state of the equalizer's vector respectively, $\underline{y}[n]=(y[n] \cdots y[n-N+1])^{\mathrm{T}}, N$ is the equalizer's tap length and $\tilde{z}[n]=z[n]-E[z[n]]$. The operator ()$^{\mathrm{T}}$ denotes for transpose of the function () and the real part of $\frac{\partial F[n]}{\partial \tilde{z}[n]}$ is a polynomial function of order three of $\tilde{z}[n]$. In this paper, the ISI is used as a measure of equalization performance and is defined by:

$$
I S I=\frac{\sum_{\tilde{m}}\left|\tilde{s}_{\tilde{m}}\right|^{2}-|\tilde{s}|_{\max }^{2}}{|\tilde{S}|_{\max }^{2}}
$$

where $|\tilde{s}|_{\max }$ is the component of $\tilde{s}$, given by $\tilde{s}=c[n] * h[n]$, having the maximal absolute value.

According to [3], the residual ISI expressed in $\mathrm{dB}$ units may be written for biased input signals as:

$$
I S I_{d B}=10 \log _{10}\left(2 m_{p}\right)-10 \log _{10}\left(E\left[|x[n]|^{2}\right]\right)
$$

where $|(\cdot)|$ is the absolute value of $(\cdot)$ and $m_{p}$ is defined by:

$$
\begin{aligned}
& \text { for } \operatorname{Sol}_{1}^{m_{p_{1}}}>0 \text { and } \operatorname{Sol}_{2}^{m_{p_{1}}}>0 \\
& m_{p}=\min \left[\operatorname{Sol}_{1}^{m_{p_{1}}}, \operatorname{Sol}_{2}^{m_{p_{1}}}\right] \\
& \text { or for } \mathrm{Sol}_{1}^{m_{p_{1}}} \cdot \mathrm{Sol}_{2}^{m_{p_{1}}}<0 \\
& m_{p}=\max \left[\mathrm{Sol}_{1}^{m_{p_{1}}}, \mathrm{Sol}_{2}^{m_{p_{1}}}\right]
\end{aligned}
$$

and 


$$
\begin{aligned}
& \operatorname{Sol}_{1}^{m_{p 1}}=\frac{-B_{1}+\sqrt{B_{1}^{2}-4 A_{1} C_{1} B}}{2 A_{1}} \\
& \operatorname{Sol}_{2}^{m_{p 1}}=\frac{-B_{1}-\sqrt{B_{1}^{2}-4 A_{1} C_{1} B}}{2 A_{1}}
\end{aligned}
$$

where

$$
\begin{aligned}
& A_{1}=B\left(45 \sigma_{\tilde{x}_{r}}^{2} a_{3}^{2}+18 \sigma_{\tilde{x}_{r}}^{2} a_{3} a_{12}+6 a_{1} a_{3}+9 \sigma_{\tilde{x}_{r}}^{2} a_{12}^{2}+2 a_{1} a_{12}\right) \\
& -2\left(3 a_{3}+a_{12}\right)+B\left(45 a_{3}^{2}+18 a_{3} a_{12}+9 a_{12}^{2}\right) \sigma_{\tilde{w}_{r}}^{2} \\
& B_{1}=B\left(12\left(\sigma_{\tilde{x}_{r}}^{2}\right)^{2} a_{3} a_{12}+6\left(\sigma_{\tilde{x}_{r}}^{2}\right)^{2} a_{12}^{2}+12 \sigma_{\tilde{x}_{r}}^{2} a_{1} a_{3}+4 \sigma_{\tilde{x}_{r}}^{2} a_{1} a_{12}+a_{1}^{2}+15 E\left[\tilde{x}_{r}^{4}\right] a_{3}^{2}+2 E\left[\tilde{x}_{r}^{4}\right] a_{3} a_{12}\right. \\
& \left.+E\left[\tilde{x}_{r}^{4}\right] a_{12}^{2}\right)-2\left(a_{1}+3 \sigma_{\tilde{x}_{r}}^{2} a_{3}+\sigma_{\tilde{x}_{r}}^{2} a_{12}\right)+B\left[45 a_{3}^{2}+18 a_{3} a_{12}+9 a_{12}^{2}\right] \sigma_{\tilde{w}_{r}}^{4} \\
& +\left(B\left(90 a_{3}^{2} \sigma_{\tilde{x}_{r}}^{2}+36 a_{3} a_{12} \sigma_{\tilde{x}_{r}}^{2}+12 a_{1} a_{3}+18 a_{12}^{2} \sigma_{\tilde{x}_{r}}^{2}+4 a_{1} a_{12}\right)-2 a_{12}-6 a_{3}\right) \sigma_{\tilde{w}_{r}}^{2} \\
& C_{1}=\left(2\left(\sigma_{\tilde{x}_{r}}^{2}\right)^{2} a_{1} a_{12}+\sigma_{\tilde{x}_{r}}^{2} a_{1}^{2}+2 E\left[\tilde{x}_{r}^{4}\right] \sigma_{\tilde{x}_{r}}^{2} a_{3} a_{12}+E\left[\tilde{x}_{r}^{4}\right] \sigma_{\tilde{x}_{r}}^{2} a_{12}^{2}+2 E\left[\tilde{x}_{r}^{4}\right] a_{1} a_{3}+E\left[\tilde{x}_{r}^{6}\right] a_{3}^{2}\right) \\
& +\left(15 a_{3}^{2}+6 a_{3} a_{12}+3 a_{12}^{2}\right) \sigma_{\tilde{w}_{r}}^{6}+\left(45 a_{3}^{2} \sigma_{\tilde{x}_{r}}^{2}+18 a_{3} a_{12} \sigma_{\tilde{x}_{r}}^{2}+6 a_{1} a_{3}+9 a_{12}^{2} \sigma_{\tilde{x}_{r}}^{2}+2 a_{1} a_{12}\right) \sigma_{\tilde{w}_{r}}^{4} \\
& +\left(a_{1}^{2}+12 a_{1} a_{3} \sigma_{\tilde{x}_{r}}^{2}+4 a_{1} a_{12} \sigma_{\tilde{x}_{r}}^{2}+15 a_{3}^{2} E\left[\tilde{x}_{r}^{4}\right]+12 a_{3} a_{12}\left(\sigma_{\tilde{x}_{r}}^{2}\right)^{2}\right. \\
& \left.+2 a_{3} a_{12} E\left[\tilde{x}_{r}^{4}\right]+a_{12}^{2} E\left[\tilde{x}_{r}^{4}\right]+6 a_{12}^{2}\left(\sigma_{\tilde{x}_{r}}^{2}\right)^{2}\right) \sigma_{\tilde{w}_{r}}^{2} \\
& \sigma_{\tilde{w}_{r}}^{2} \simeq 0.5 \frac{E\left[|x[n]|^{2}\right]}{S N R \sum_{k=0}^{R-1}|h[k]|^{2}} \\
& B \simeq \mu N\left(\sigma_{\tilde{x}}^{2}+|E[x[n]]|^{2}\right) \sum_{k=0}^{R-1}|h[k]|^{2}+\mu \frac{E\left[|x[n]|^{2}\right]}{S N R} N
\end{aligned}
$$

$R$ is the channel length, $a_{1}, a_{12}, a_{3}$ are properties of the chosen equalizer and found by:

$$
\operatorname{Re}\left(\frac{\partial F[n]}{\partial \tilde{z}[n]}\right)=\left(a_{1}\left(\tilde{z}_{r}\right)+a_{3}\left(\tilde{z}_{r}\right)^{3}+a_{12}\left(\tilde{z}_{r}\right)\left(\tilde{z}_{i}\right)^{2}\right)
$$

where $\operatorname{Re}(\cdot)$ is the real part of $(\cdot)$ and $\tilde{z}_{r}, \tilde{z}_{i}$ are the real and imaginary parts of $\tilde{z}[n], \sigma_{\tilde{x}_{r}}^{2}$ is the variance of $\tilde{x}_{r}[n]\left(\tilde{x}_{r}[n]\right.$ is the real part of $\left.\tilde{x}[n]\right), \sigma_{\tilde{x}}^{2}$ is the variance of $\tilde{x}[n]$ and $S N R$ is given by:

$$
S N R=\frac{E|x[n]|^{2}}{\sigma_{w}^{2}}
$$

Please note that (5) can be also applied for the non-biased case by substituting $E[x[n]]=0$. For biased input signals we have that $E|x[n]|^{2}$ is higher than for the non-biased input signal case. Thus, it is reasonable to think according to (5) that improved equalization performance may be obtained from the residual ISI point of view for biased input signals compared to the non-biased version. But, on the other hand, the expression for $m_{p}$ in (5) may be much higher for biased input signals compared to the non-biased case due to the bias of the input sequence (namely, $E[x[n]]$ ) in the expression of $B$ (12) which causes $B \quad(12)$ to be higher for biased input signals compared to the non-biased case. Please note that $B(12)$ has a direct impact also on $A_{1}(8)$ and $B_{1}$ (9). Thus, from (5) it is unclear if improved or degraded equalization performance is obtained in the residual ISI point of view for biased input signal case compared to the non-biased version. 


\section{Condition for Improved Equalization Performance}

In this section, we first derive a closed-form approximated expression for the difference in the residual ISI obtained by blind adaptive equalizers with biased input signals compared to the non-biased case. Then, based on this new derived expression, we derive the condition for which improved equalization performance is obtained from the residual ISI point of view for the non-biased input case compared to the biased version. In the following we denote $I S I_{d B}, A_{1}, B_{1}, B$ and $S N R$ for the non-biased case (by substituting $E[x[n]]=0$ into (5), (8), (9), (12) and (14)) as $I S I_{n b}, A_{1_{n b}}, B_{1_{n b}}, B_{n b}$ and $S N R_{n b}$ respectively. In addition, for the biased case, we denote (5), (8), (9), (12) and (14) as $I S I_{b}, A_{1_{b}}, B_{1_{b}}, B_{b}$ and $S N R_{b}$ respectively. To facilitate reading, we denote $E[x[n]]$ as $m$.

\section{Theorem:}

$$
\begin{aligned}
& I S I_{b}=I S I_{n b}+10 \log _{10}\left(\frac{1+\frac{b}{B_{n b}}}{\left(1+\frac{b b}{B_{1_{n b}}}\right)\left(1+\frac{|m|^{2}}{\sigma_{\tilde{x}}^{2}}\right)}\right) \Downarrow \\
& \Delta I S I=10 \log _{10}\left(\frac{1+\frac{b}{B_{n b}}}{\left(1+\frac{b b}{B_{1_{n b}}}\right)\left(1+\frac{|m|^{2}}{\sigma_{\tilde{x}}^{2}}\right)}\right)
\end{aligned}
$$

where $\Delta I S I$ is the closed-form approximated expression for the difference in the residual ISI obtained by blind adaptive equalizers with biased input signals compared to the non-biased case and

$$
\begin{gathered}
\quad b=\mu N|m|^{2} \sum_{k=0}^{R-1}|h[k]|^{2} \\
b b=b\left(a+r \sigma_{\tilde{x}_{r}}^{4}+q \sigma_{\tilde{x}_{r}}^{2}\right) \\
a=12\left(\sigma_{\tilde{x}_{r}}^{2}\right)^{2} a_{3} a_{12}+6\left(\sigma_{\tilde{x}_{r}}^{2}\right)^{2} a_{12}^{2}+12 \sigma_{\tilde{x}_{r}}^{2} a_{1} a_{3}+4 \sigma_{\tilde{x}_{r}}^{2} a_{1} a_{12}+a_{1}^{2} \\
+15 E\left[\tilde{x}_{r}^{4}\right] a_{3}^{2}+2 E\left[\tilde{x}_{r}^{4}\right] a_{3} a_{12}+E\left[\tilde{x}_{r}^{4}\right] a_{12}^{2} \\
r=45 a_{3}^{2}+18 a_{3} a_{12}+9 a_{12}^{2} \\
q=90 a_{3}^{2} \sigma_{\tilde{x}_{r}}^{2}+36 a_{3} a_{12} \sigma_{\tilde{x}_{r}}^{2}+12 a_{1} a_{3}+18 a_{12}^{2} \sigma_{\tilde{x}_{r}}^{2}+4 a_{1} a_{12}
\end{gathered}
$$

Proof:

Based on (14) we have:

$$
S N R_{b}=\frac{\sigma_{\tilde{x}}^{2}+|m|^{2}}{\sigma_{w}^{2}}=\frac{\sigma_{\tilde{x}}^{2}}{\sigma_{w}^{2}}\left(1+\frac{|m|^{2}}{\sigma_{\tilde{x}}^{2}}\right)=S N R_{n b}\left(1+\frac{|m|^{2}}{\sigma_{\tilde{x}}^{2}}\right)
$$

With the help of (11) and (18) we may write:

$$
\begin{aligned}
\sigma_{\tilde{\psi}_{r}}^{2} & =\frac{1}{2} \frac{\left(\sigma_{\tilde{x}}^{2}+|m|^{2}\right)}{S N R_{b} \sum_{k=0}^{R-1}|h[k]|^{2}}=\frac{1}{2}\left(1+\frac{|m|^{2}}{\sigma_{\tilde{x}}^{2}}\right) \frac{\sigma_{\tilde{x}}^{2}}{S N R_{n b}\left(1+\frac{|m|^{2}}{\sigma_{\tilde{x}}^{2}} \sum_{k=0}^{R-1}|h[k]|^{2}\right.} \\
\sigma_{\tilde{\psi}_{r}}^{2} & =\frac{1}{2} \frac{\sigma_{\tilde{x}}^{2}}{S N R_{n b} \sum_{k=0}^{R-1}|h[k]|^{2}}=\frac{\sigma_{\tilde{x}_{r}}^{2}}{S N R_{n b} \sum_{k=0}^{R-1}|h[k]|^{2}}
\end{aligned}
$$


From (19) we may conclude that $C_{1}(10)$ has the same value for the biased and non-biased input case. Next, we wish to find the relationship between $B_{b}$ and $B_{n b}$ and between $B_{1_{b}}$ and $B_{1_{n b}}$. For that purpose we use (12) and (18) to obtain:

$$
B_{b}=\mu N\left(\sigma_{\tilde{x}}^{2}+|m|^{2}\right) \sum_{k=0}^{R-1}|h[k]|^{2}+\frac{\mu N\left(\sigma_{\tilde{x}}^{2}+|m|^{2}\right)}{S N R_{b}}=\mu N \sigma_{\tilde{x}}^{2}\left(1+\frac{|m|^{2}}{\sigma_{\tilde{x}}^{2}}\right) \sum_{k=0}^{R-1}|h[k]|^{2}+\frac{\mu N \sigma_{\tilde{x}}^{2}\left(1+\frac{|m|^{2}}{\sigma_{\tilde{x}}^{2}}\right)}{S N R_{n b}\left(1+\frac{|m|^{2}}{\sigma_{\tilde{x}}^{2}}\right)}
$$

From (20) we have:

$$
B_{b}=\mu N \sigma_{\tilde{x}}^{2}\left(1+\frac{|m|^{2}}{\sigma_{\tilde{x}}^{2}}\right) \sum_{k=0}^{R-1}|h[k]|^{2}+\frac{\mu N \sigma_{\tilde{x}}^{2}}{S N R_{n b}}
$$

Next by substituting $m=0$ into (12) and using (18), we may write (21) as:

$$
B_{b}=B_{n b}+\mu N|m|^{2} \sum_{k=0}^{R-1}|h[k]|^{2}=B_{n b}+b
$$

where

$$
B_{n b}=\mu N \sigma_{\tilde{x}}^{2} \sum_{k=0}^{R-1}|h[k]|^{2}+\frac{\mu N \sigma_{\tilde{x}}^{2}}{S N R_{n b}}
$$

and $b$ is given in (16). Based on (22) we may write:

$$
B_{b}=B_{n b}\left(1+\frac{b}{B_{n b}}\right)
$$

Next we turn to find the relationship between $B_{1_{b}}$ and $B_{1_{n b}}$. From (9) we may write:

$$
B_{1_{b}}=B_{b} a-s+B_{b} r \sigma_{\tilde{w}_{r}}^{4}+\left(B_{b} q-d\right) \sigma_{\tilde{w}_{r}}^{2}
$$

where $a, r$ and $q$ are given in (17) and

$$
\begin{aligned}
& s=2\left(a_{1}+3 \sigma_{\tilde{x}_{r}}^{2} a_{3}+\sigma_{\tilde{x}_{r}}^{2} a_{12}\right) \\
& d=2 a_{12}+6 a_{3}
\end{aligned}
$$

From (25) and (24) we may have:

$$
\begin{aligned}
B_{1_{b}} & =B_{b}\left(a+r \sigma_{\tilde{w}_{r}}^{4}+q \sigma_{\tilde{w}_{r}}^{2}\right)-d \sigma_{\tilde{w}_{r}}^{2}-s \\
& =B_{n b}\left(1+\frac{b}{B_{n b}}\right)\left(a+r \sigma_{\tilde{w}_{r}}^{4}+q \sigma_{\tilde{w}_{r}}^{2}\right)-d \sigma_{\tilde{w}_{r}}^{2}-s
\end{aligned}
$$

which can be also written as:

$$
B_{1_{b}}=B_{1_{n b}}+b\left(a+r \sigma_{\tilde{w}_{r}}^{4}+q \sigma_{\tilde{w}_{r}}^{2}\right)=B_{1_{n b}}+b b=B_{1_{n b}}\left(1+\frac{b b}{B_{1_{n b}}}\right)
$$

where $b b$ is given in (17) and $B_{1_{n b}}$ is obtained by substituting $m=0$ into (9):

$$
B_{1_{n b}}=B_{n b}\left(a+r \sigma_{\tilde{w}_{r}}^{4}+q \sigma_{\tilde{w}_{r}}^{2}\right)-d \sigma_{\tilde{w}_{r}}^{2}-s
$$

The solution for $m_{p}$ given in (6) is acually based on the following second order equation with respect to $m_{p}[15]:$

$$
A_{1} m_{p}^{2}+B_{1} m_{p}+B C_{1}=0
$$


According to [15], $m_{p}$ may be very small in the convergence state so that the part of $A_{1} m_{p}^{2}$ may be neglectable compared to $B_{1} m_{p}+B C_{1}$. If this is the case then the solution for $m_{p}$ is:

$$
m_{p}=-\frac{B C_{1}}{B_{1}}
$$

Simulation results carried out in [15] have shown that better accuracy is obtained when using (6) over (31) for the non-biased case which is not surprising. But, the difference in the accuracy is not so high making the solution for $m_{p}$ given in (31) acceptable. In the following we denote $m_{p}$ as $m_{p_{b}}$ and $m_{p_{n b}}$ for the biased and non-biased input case respectivaly. By using (31), (28) and (24) we may have:

$$
m_{p_{b}}=-\frac{B_{b} C_{1}}{B_{1 b}}=-\frac{B_{n b}\left(1+\frac{b}{B_{n b}}\right) C_{1}}{B_{1_{b}}}=-\frac{B_{n b}\left(1+\frac{b}{B_{n b}}\right) C_{1}}{B_{1_{n b}}\left(1+\frac{b b}{B_{1_{n b}}}\right)}=-\frac{B_{n b} C_{1}}{B_{1_{n b}}} \frac{\left(1+\frac{b}{B_{n b}}\right)}{\left(1+\frac{b b}{B_{1_{n b}}}\right)}=m_{p_{n b}} \frac{\left(1+\frac{b}{B_{n b}}\right)}{\left(1+\frac{b b}{B_{1_{n b}}}\right)}
$$

where

$$
m_{p_{n b}}=-\frac{B_{n b} C_{1}}{B_{1_{n b}}}
$$

Next, by using (5) we may have:

$$
\begin{aligned}
I S I_{b} & =10 \log _{10}\left(2 m_{p_{b}}\right)-10 \log _{10}\left(\sigma_{\tilde{x}}^{2}+|m|^{2}\right) \\
& =10 \log _{10}\left(2 m_{p_{b}}\right)-10 \log _{10}\left(\sigma_{\tilde{x}}^{2}\left(1+\frac{|m|^{2}}{\sigma_{\tilde{x}}^{2}}\right)\right) \\
& =10 \log _{10}\left(2 m_{p_{b}}\right)-10 \log _{10}\left(\sigma_{\tilde{x}}^{2}\right)-10 \log _{10}\left(1+\frac{|m|^{2}}{\sigma_{\tilde{x}}^{2}}\right)
\end{aligned}
$$

Now we substitute (32) into (34) and obtain:

$$
\begin{aligned}
I S I_{b} & =10 \log _{10}\left(2 m_{p_{n b}} \frac{\left(1+\frac{b}{B_{n b}}\right)}{\left(1+\frac{b b}{B_{1_{n b}}}\right)}\right)-10 \log _{10}\left(\sigma_{\tilde{x}}^{2}\right)-10 \log _{10}\left(1+\frac{|m|^{2}}{\sigma_{\tilde{x}}^{2}}\right) \\
& =10 \log _{10}\left(2 m_{p_{n b}}\right)+10 \log _{10}\left(\frac{1+\frac{b}{B_{n b}}}{1+\frac{b b}{B_{1_{n b}}}}\right)-10 \log _{10}\left(\sigma_{\tilde{x}}^{2}\right)-10 \log _{10}\left(1+\frac{|m|^{2}}{\sigma_{\tilde{x}}^{2}}\right) \\
& =10 \log _{10}\left(2 m_{p_{n b}}\right)-10 \log _{10}\left(\sigma_{\tilde{x}}^{2}\right)+10 \log _{10}\left(\frac{1+\frac{b}{B_{n b}}}{1+\frac{b b}{B_{1_{n b}}}}\right)-10 \log _{10}\left(1+\frac{|m|^{2}}{\sigma_{\tilde{x}}^{2}}\right) \\
& =I S I_{n b}+10 \log _{10}\left(\frac{1+\frac{b}{B_{n b}}}{1+\frac{b b}{B_{1_{n b}}}}\right)-10 \log _{10}\left(1+\frac{|m|^{2}}{\sigma_{\tilde{x}}^{2}}\right)=I S I_{n b}+10 \log _{10}\left(\frac{1+\frac{b}{B_{n b}}}{\left(1+\frac{b b}{B_{1_{n b}}}\right)\left(1+\frac{|m|^{2}}{\sigma_{\tilde{x}}^{2}}\right)}\right)
\end{aligned}
$$


where

$$
I S I_{n b}=10 \log _{10}\left(2 m_{p_{n b}}\right)-10 \log _{10}\left(\sigma_{\tilde{x}}^{2}\right)
$$

This completes our proof.

$I S I_{b}$ as well as $I S I_{n b}$ (15) hold negative values. Perfect equalization performance in the residual ISI point of view is achieved for the biased case if $I S I_{b} \rightarrow-\infty$. In addition, perfect equalization performance in the residual ISI point of view is achieved for the non-biased case if $I S I_{n b} \rightarrow-\infty$. Thus, if $\Delta I S I$ (15) is positive $(\Delta I S I>0)$ it implies that improved equalization performance is obtained in the residual ISI point of view for the non-biased case compared with the biased version. In other words, according to (15), if

$$
1+\frac{b}{B_{n b}}>\left(1+\frac{b b}{B_{1_{n b}}}\right)\left(1+\frac{|m|^{2}}{\sigma_{\tilde{x}}^{2}}\right)
$$

then improved equalization performance is obtained from the residual ISI point of view for the non-biased input case compared to the biased version. In the following we will show the relationship between $\frac{b}{B_{n b}}$ and $\frac{|m|^{2}}{\sigma_{\tilde{x}}^{2}}$. By using (16) and (23) we have:

$$
\frac{b}{B_{n b}}=\frac{\mu N|m|^{2} \sum_{k=0}^{R-1}|h[k]|^{2}}{\mu N \sigma_{\tilde{x}}^{2} \sum_{k=0}^{R-1}|h[k]|^{2}+\frac{\mu N \sigma_{\tilde{x}}^{2}}{S N R_{n b}}}=\frac{|m|^{2}}{\sigma_{\tilde{x}}^{2}} \frac{\sum_{k=0}^{R-1}|h[k]|^{2}}{\sum_{k=0}^{R-1}|h[k]|^{2}+\frac{1}{S N R_{n b}}} \leq \frac{|m|^{2}}{\sigma_{\tilde{x}}^{2}}
$$

Thus, according to (38), we may conclude that if $\frac{b b}{B_{1_{n b}}}>0$ then improved equalization performance is obtained from the residual ISI point of view for the biased input case compared to the non-biased version. Please note that (37) depends on the step-size parameter, equalizer's tap length, input signal statistics, channel power, signal to noise ratio and on the properties of the chosen blind equalizer via $a_{1}, a_{12}$ and $a_{3}$ from (13).

\section{Simulation}

In this section, $\Delta I S I$ (15) was tested via simulation by using Godard's algorithm [17]. Please note that if $\Delta I S I>0$ then improved equalization performance is obtained in the residual ISI point of view for the nonbiased input case compared with the biased version. The equalizer's taps for Godard's algorithm [17] were updated according to:

$$
c_{m}[n+1]=c_{m}[n]-\mu_{G}\left(|z[n]-E[x[n]]|^{2}-\frac{E\left[|x[n]-E[x[n]]|^{4}\right]}{E\left[|x[n]-E[x[n]]|^{2}\right]}\right)(z[n]-E[x[n]]) y^{*}[n-m]
$$

where, $\mu_{G}$ is the step-size. The values for $a_{1}, a_{12}$ and $a_{3}$ corresponding to Godard's [17] algorithm are defined as $a_{1}^{G}, a_{12}^{G}$ and $a_{3}^{G}$ respectively and are given by:

$$
a_{1}^{G}=-\frac{E\left[\mid x[n]-E[x[n]]^{4}\right]}{E\left[\mid x[n]-E[x[n]]^{2}\right]} ; \quad a_{12}^{G}=1 ; \quad a_{3}^{G}=1
$$

A biased 16QAM, a modulation using $\pm\{1,3\}$ levels for in-phase and quadrature components in addition to a given bias was considered. The bias for the real and imaginary axes were the same. In our simulation we used the channel given in [18]: $h_{n}=\left(0\right.$ for $n<0 ;-0.4$ for $n=0 ; 0.84 \cdot 0.4^{n-1}$ for $\left.n>0\right)$.

Figures 2-5 are the simulated performance of (39) for the biased 16QAM input case, namely the ISI as a function of iteration number for various SNR values and two different biases, compared with the non-biased case. Figures 6-9 are the zoomed versions of Figures 2-5 respectively. According to Figure 6 the simulated 


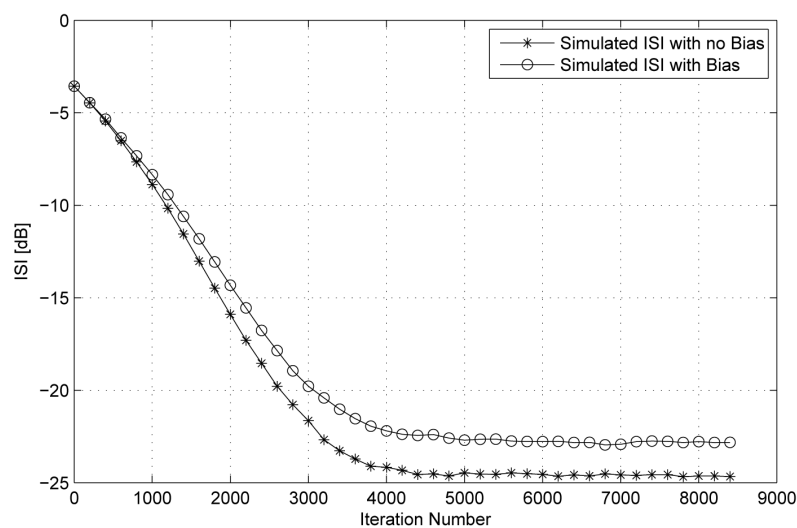

Figure 2. Simulated ISI performance comparison between the biased 16QAM input case and the non-biased version using (39) for $S N R_{b}=30[\mathrm{~dB}]$. The averaged results were obtained in 100 Monte Carlo trials. $N=13$ and $\mu_{G}=17 \times 10^{-6}$. The bias was set to $6+6 j$.

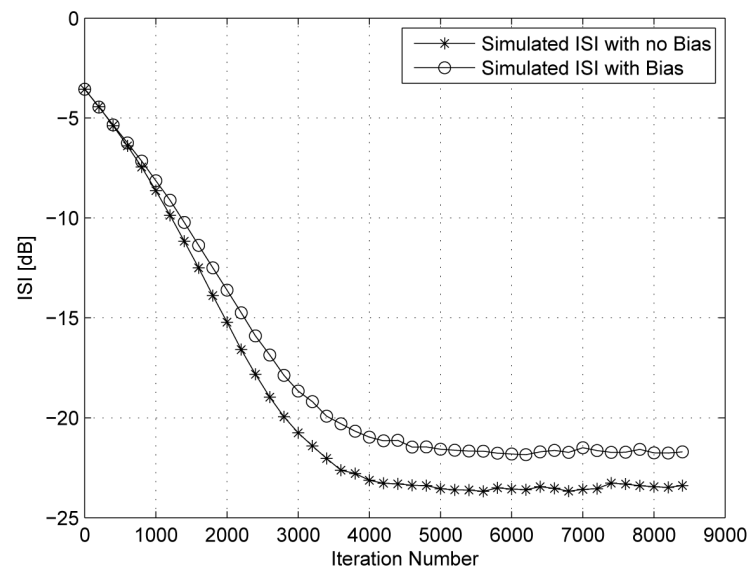

Figure 3. Simulated ISI performance comparison between the biased 16QAM input case and the non-biased version using (39) for $S N R_{b}=25[\mathrm{~dB}]$. The averaged results were obtained in 100 Monte Carlo trials. $N=13$ and $\mu_{G}=17 \times 10^{-6}$. The bias was set to $6+6 j$.

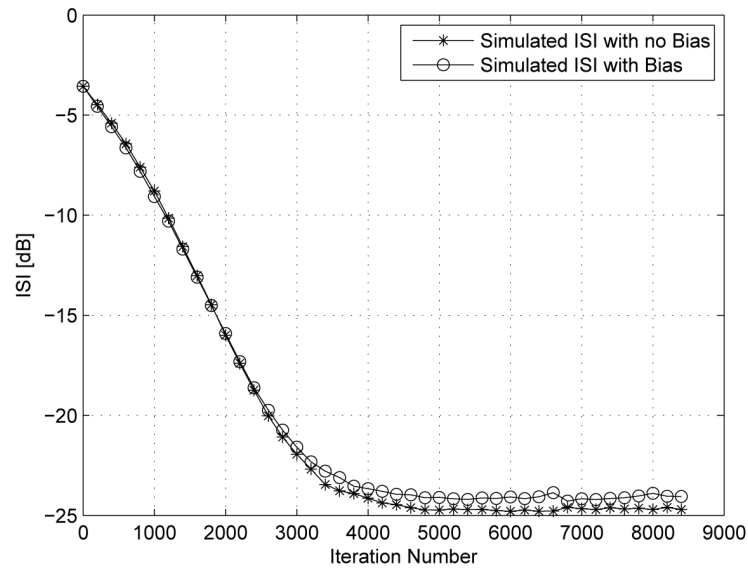

Figure 4. Simulated ISI performance comparison between the biased 16QAM input case and the non-biased version using (39) for $S N R_{b}=30[\mathrm{~dB}]$. The averaged results were obtained in 100 Monte Carlo trials. $N=13$ and $\mu_{G}=17 \times 10^{-6}$. The bias was set to $4+4 j$. 


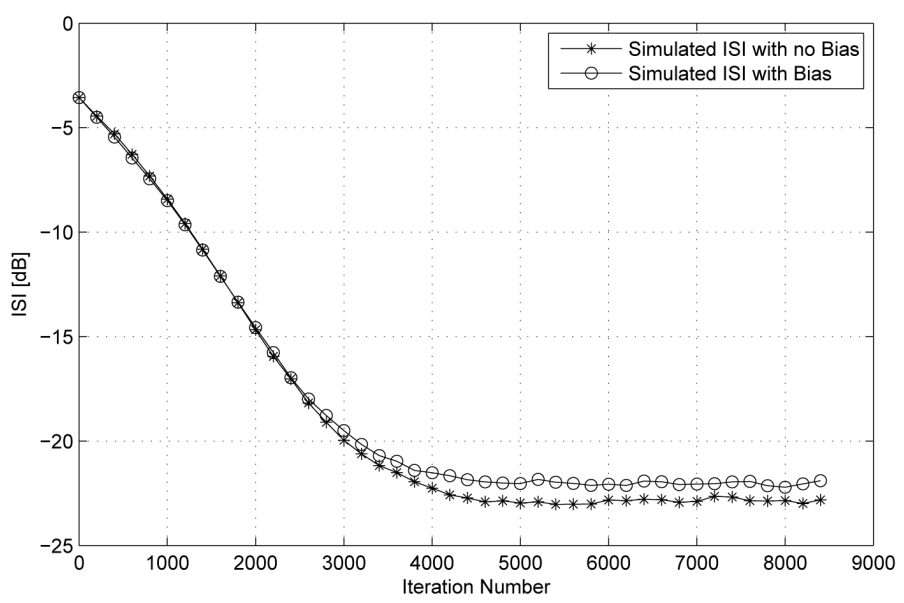

Figure 5. Simulated ISI performance comparison between the biased 16QAM input case and the non-biased version using (39) for $S N R_{b}=20[\mathrm{~dB}]$. The averaged results were obtained in 100 Monte Carlo trials. $N=13$ and $\mu_{G}=17 \times 10^{-6}$. The bias was set to $4+4 j$.

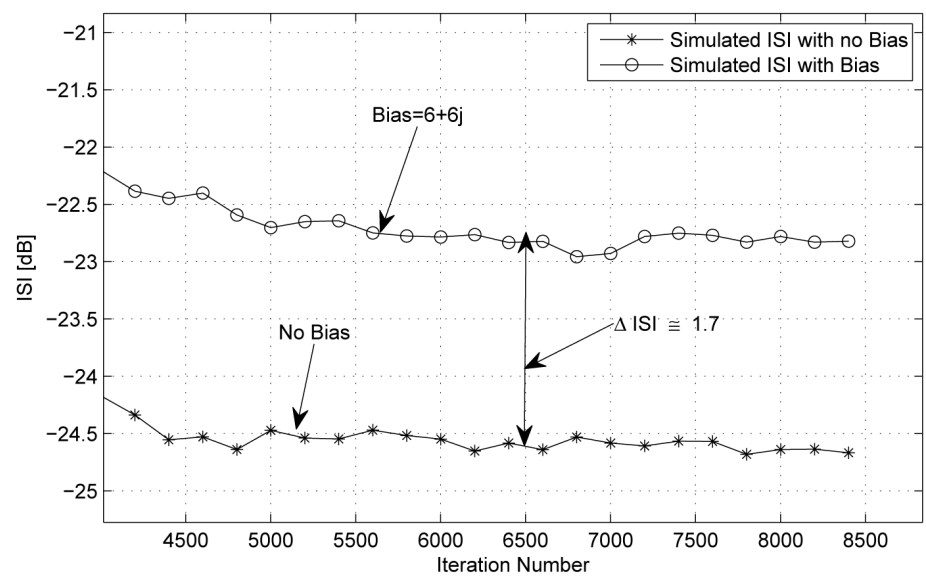

Figure 6. Zoomed version of Figure 2.

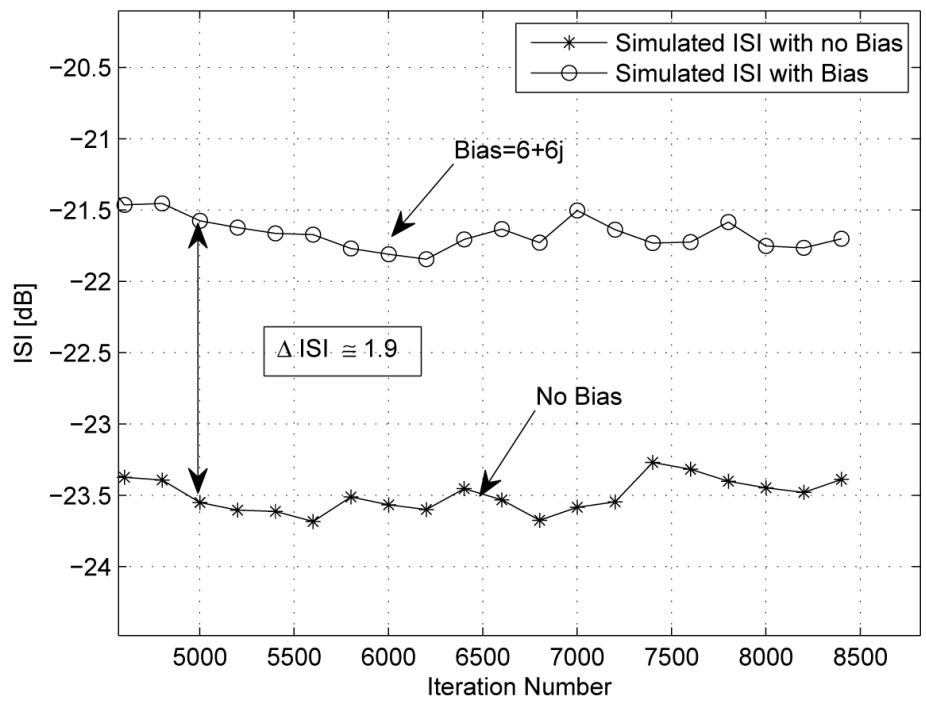

Figure 7. Zoomed version of Figure 3. 


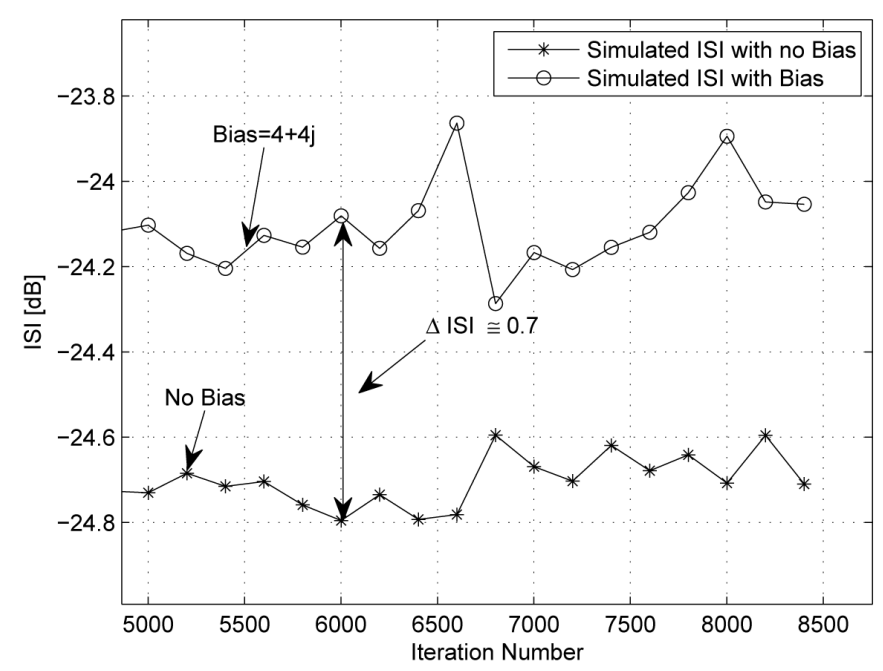

Figure 8. Zoomed version of Figure 4.

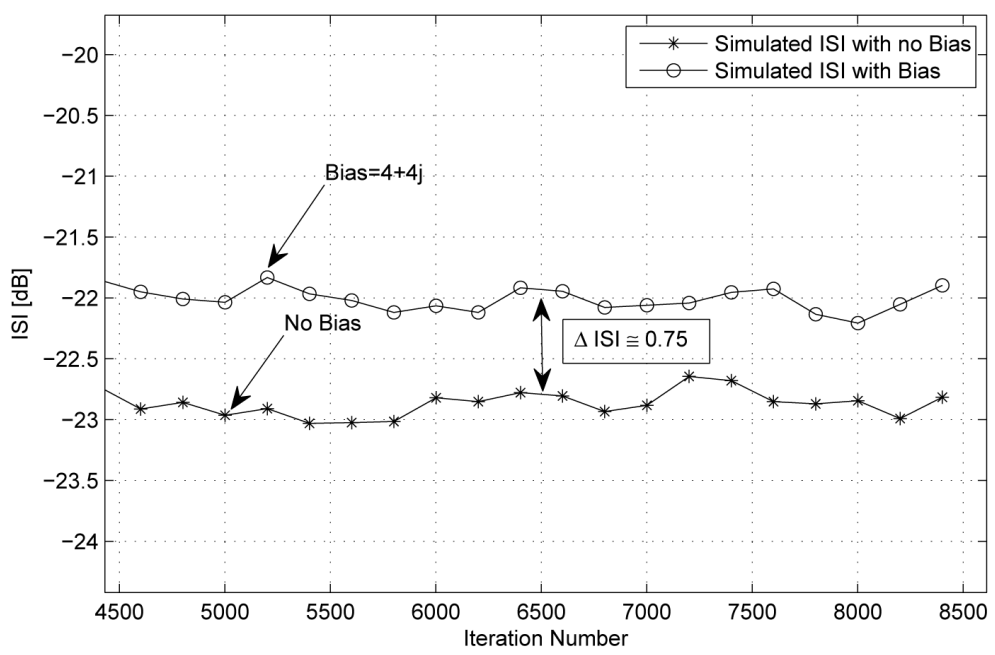

Figure 9. Zoomed version of Figure 5.

difference in the residual ISI between the biased and non-biased case is $\Delta I S I \cong 1.7$ while according to (15) we have $\Delta I S I \cong 1.92$.

According to Figure 7 the simulated difference in the residual ISI between the biased and non-biased case is $\Delta I S I \cong 1.9$ while according to (15) we have $\Delta I S I \cong 2.102$.

According to Figure 8 the simulated difference in the residual ISI between the biased and non-biased case is $\Delta I S I \cong 0.7$ while according to (15) we have $\Delta I S I \cong 0.73$.

According to Figure 9 the simulated difference in the residual ISI between the biased and non-biased case is $\Delta I S I \cong 0.75$ while according to (15) we have $\Delta I S I \cong 0.77$.

Based on Figures 6-9, the simulated results for $\Delta I S I$ and those results obtained from (15) for $\Delta I S I$ are very close. Thus, the expression for $\Delta I S I$ (15) is accurate enough for saying that if $\Delta I S I>0$ then improved equalization performance is obtained in the residual ISI point of view for the non-biased input case compared to the biased version. Please note that according to Figures 2-5, improved equalization performance was obtained in the residual ISI point of view for the non-biased input case compared to the biased version which was also confirmed by the expression for $\Delta I S I$ (15) since $\Delta I S I$ was found to be positive for all the mentioned cases.

\section{Conclusion}

In this paper, we derived for the real and two independent quadrature carrier case a closed-form approximated 
expression for the difference in the residual ISI obtained by blind adaptive equalizers with biased input signals compared to the non-biased case. This expression depends on the step-size parameter, equalizer's tap length, input signal statistics, channel power, SNR and chosen equalizer via $a_{1}, a_{12}$ and $a_{3}$. It is applicable for blind adaptive equalizers where the error fed into the adaptive mechanism, which updates the equalizer's taps, can be expressed as a polynomial function of order three of the equalized output and where the gain between the input and equalized output signal is equal to one as is in the case of Godard's algorithm. Based on this expression, we have shown under what condition improved equalization performance is obtained from the residual ISI point of view for the non-biased case compared with the biased version.

\section{Acknowledgements}

We thank the editor and the referee for their comments.

\section{References}

[1] Pinchas, M. (2010) A New Closed Approximated Formed Expression for the Achievable Residual ISI Obtained by Adaptive Blind Equalizers for the Noisy Case. IEEE International Conference on Wireless Communications, Networking and Information Security (WCNIS), Beijing, 25-27 June 2010, 26-30. http://dx.doi.org/10.1109/WCINS.2010.5541879

[2] Pinchas, M. (2013) Residual ISI Obtained by Blind Adaptive Equalizers and Fractional Noise. Mathematical Problems in Engineering, 2013, 1-11. http://dx.doi.org/10.1155/2013/972174

[3] Panziel, N. and Pinchas, M. (2014) An Approximated Expression for the Residual ISI Obtained by Blind Adaptive Equalizer and Biased Input Signals. Journal of Signal and Information Processing (JSIP), 5, 155-178.

[4] Haykin, S. (1991) Blind Deconvolution. In: Haykin, S., Ed., Adaptive Filter Theory, Chapter 20, Prentice-Hall, Englewood Cliffs.

[5] Yang, D.H., Li, G. and Zhu, Z.H. (2011) A Novel Structure for Adaptive Blind Channel Equalization. 7th International Conference on Wireless Communications, Networking and Mobile Computing (WiCOM), Wuhan, 23-25 September 2011, 1-4. http://dx.doi.org/10.1109/wicom.2011.6039953

[6] Feng, C. and Chi, C. (1999) Performance of Cumulant Based Inverse Filters for Blind Deconvolution. IEEE Transaction on Signal Processing, 47, 1922-1936. http://dx.doi.org/10.1109/78.771041

[7] Wen, S.-Y. and Liu, F. (2010) A Computationally Efficient Multi-Modulus Blind Equalization Algorithm. The 2nd IEEE International Conference on Information Management and Engineering (ICIME), Chengdu, 16-18 April 2010, 685-687. http://dx.doi.org/10.1109/ICIME.2010.5478261

[8] He, N. (2010) Application of Adaptive Equalizer in Digital Microwave Communication. International Conference on Electronics and Information Engineering (ICEIE), 2, 497-500. http://dx.doi.org/10.1109/ICEIE.2010.5559759

[9] Sheikh, S.A. and Fan, P.Z. (2008) New Blind Equalization Techniques Based on Improved Square Contour Algorithm. Digital Signal Processing, 18, 680-693. http://dx.doi.org/10.1016/j.dsp.2007.09.001

[10] Sato, Y. (1975) A Method of Self-Recovering Equalization for Multilevel Amplitude Modulation. IEEE Transactions on Communications, 23, 679-682. http://dx.doi.org/10.1109/TCOM.1975.1092854

[11] Tuğcu, E., Çakır, F. and Ozen, A. (2013) A New Step Size Control Technique for Blind and Non-Blind Equalization Algorithms. Radioengineering, 22, 4-51.

[12] Liu, Z. and Ning, X.L. (2012) Comparison of Equalization Algorithms for Underwater Acoustic Channels. The 2nd International Conference on Computer Science and Network Technology, Changchun, 29-31 December 2012, $2059-2063$. http://dx.doi.org/10.1109/ICCSNT.2012.6526324

[13] Vanka, R.N., Murty, S.B. and Mouli, B.C. (2014) Performance Comparison of Supervised and Unsupervised/Blind Equalization Algorithms for QAM Transmitted Constellations. 2014 International Conference on Signal Processing and Integrated Networks (SPIN), Noida, 20-21 February 2014, 316-321. http://dx.doi.org/10.1109/SPIN.2014.6776970

[14] Qin, Q., Li, H.H. and Jiang, T.Y. (2013) A New Study on VCMA-Based Blind Equalization for Underwater Acoustic Communications. 2013 International Conference on Mechatronic Sciences, Electric Engineering and Computer (MEC), Shenyang, 20-22 December 2013, 3526-3529.

[15] Pinchas, M. (2010) A Closed Approximated Formed Expression for the Achievable Residual Intersymbol Interference Obtained by Blind Equalizers. Signal Processing, 90, 1940-1962. http://dx.doi.org/10.1016/j.sigpro.2009.12.014

[16] Kupchan, S. and Pinchas, M. (2014) A Closed-Form Approximated Expression for the Residual ISI Obtained by Blind 
Adaptive Equalizers with Gain Equal or Less than One. Radioengineering, 23, 954.

[17] Godard, D.N. (1980) Self Recovering Equalization and Carrier Tracking in Two-Dimensional Data Communication System. IEEE Transactions on Communications, 28, 1867-1875. http://dx.doi.org/10.1109/TCOM.1980.1094608

[18] Shalvi, O. and Weinstein, E. (1990) New Criteria for Blind Deconvolution of Nonminimum Phase Systems (Channels). IEEE Transactions on Information Theory, 36, 312-321. http://dx.doi.org/10.1109/18.52478 